\title{
Menopause, cessation of menses, vs. "menopause”, a primary brain disorder?
}

\author{
S. L. Jaffe
}

Department of Neurology, Louisiana State University School of Medicine, Shreveport, USA

Email: sjaffe1@1suhsc.edu

Received 18 March 2013; revised 5 May 2013; accepted 20 May 2013

Copyright (C) 2013 S. L. Jaffe. This is an open access article distributed under the Creative Commons Attribution License, which permits unrestricted use, distribution, and reproduction in any medium, provided the original work is properly cited.

\section{ABSTRACT}

The "menopause" (not menopause, the cessation of menses) mechanism responsible for its symptom complex (Menopausal Symptom Complex) (or, Climacteric Syndrome) including chronic fatigue, fibromyalgia, depression, tension headache, cervical vertigo, sleep disturbances, irritability, anxiety/panic attacks, cognitive changes (decreased concentration to obsessional/delusional thought), decreased libido, and vasomotor dysfunction remains elusive. And MSC, other than vasomotor dysfunction, is not significantly altered by treatment with sex hormones (estrogen/progesterone), which have major neoplastic and vascular side effects. Thus at present, there are minimal indications for treatment with these compounds. Confusion between menopause and "menopause” (MSC) as well as research money made available by pharma advocating ERT/HRT (estrogen replacement therapy/hormone replacement therapy) has produced both therapeutic and research inertia. Presently, there would appear to be mismanagement of a symptom complex which infers primary brainlimbic system dysfunction and for which there is no correlation with falling sex hormone levels. Pharmacological modification of the proposed aberrant limbic circuitry responsible for the MSC has been successfully accomplished using specific NT/MMs (neurotransmission/modulation modifiers) i.e. a combination of alpha-1 and norepinephrine reuptake blockers, and thus providing initial elucidation of this particular brain pathophysiology as well as an efficient treatment of a problem affecting up to $60 \%$ of women ages 35 to 55. Specific NT/MMs capable of affecting a number of neurotransmitter/receptor types within limbic circuitry appear to reverse the MSC which includes chronic fatigue and fibromyalgia, pointing to aberrant limbic circuitry as their etiology as well.
Keywords: Menopause; Menopause Treatment; Brain Plasticity; Neurotransmission/Neuromodulation; Fibromyalgia/Chronic Fatigue

\section{INTRODUCTION}

Over the past 10 years, the approach to the concept of menopause has become more rationale. We have slowly isolated the objective manifestations of menopause (i.e. the permanent cessation of menstruation resulting from the loss of ovarian follicular activity), and the definitive clinical effects from this process are minimal: hot flashes and atrophic vaginitis. Only these remain an indication for ERT/ HRT [1]. And of course the latter can be treated topically; and even hot flashes appear to respond to brain NT/MMs such as fluoxetine, trazadone and venlafaxine $[2,3]$. Thus at present, there appears to be minimal indication (severe hot flashes) for even short term ERT/HRT especially with their major vascular and neoplastic side effects as well as their lack of protective effects, and in fact, often deleterious effects on cardiovascular, cerebrovascular, Alzheimer's and Parkinson's diseases [4,5]. Even osteoporosis can be more efficiently treated by other agents; and sex steroid levels have not been found to differ in post-menopausal women with and without Type I (postmenopausal) osteoporosis implicating other factors [6]. Furthermore, menopause is associated in most women with many positive effects surrounding the disappearance of the monthly "curse". So why has menopause become such a controversial, major medical issue over the past century? Because of confusion between menopause and "menopause" (i.e. the MSC), and, as often happens, funding in its various manifestations by big pharma with, for the most part, unintended collusion by the scientific/medical community.

\section{THE CONFUSION}

Major changes in female behavior associated with age- 
related menstrual dysregulation have been documented for over a century in the medical and lay literature, but the relationship between natural menopause as defined above, and the trans-cultural [7-9] cognitive, emotional and somatic symptoms most likely to bring women in this mid-life range to the physician or menopause clinic has been very unclear until recently [10]. Specifically, the symptom presentation, i.e. "menopause", referred to in this country as the MSC and in Europe as the Climacteric Syndrome, includes: 1) depression; 2) chronic fatigue; 3) fibromyalgia; 4) sleep disturbances; 5) headache (mostly tension) with/without cervical vertigo (caused by asymmetry of cervical muscle spasm); 6) irritability; 7) anxiety to frank panic attacks; 8) cognitive changes ranging from decreased concentration to obsessional and/or delusional thought ("menopausal madness"); 9) decreased libido; 10) and the hormonally related condition (at least partially) of vasomotor dysfunction i.e. hot flashes $[10,11]$. Moreover, the incidence grouping of these symptoms (beyond the last condition) is not exclusive to the time of permanent menstrual cessation or to any specific age groups in the 35 - 55 years old age range [12]. And MSC observations demonstrate the major incidence to be in the 33 - 45 years old age group.

There has been no consistent evidence to implicate a mechanistic relationship between falling estrogen/progesterone levels and the MSC, and there are major inconsistencies in the so-called "domino" as well as psychosocial theories $[10,13]$. Moreover, depression has not been significantly altered by either ERT or HRT. In fact, progesterone can increase depressive symptoms; and estrogen has no effect on clinical depression even at prohibitively high doses $[14,15]$. The evidence for acute estrogen/ progesterone effects on neuronal membranes is at best controversial, and these experimental findings may only involve secondary effects resulting from upstream sensing by the brain's hypothalamic-pituitary axis which then secondarily activates other brain circuitry. The best evidence indicates that receptors for these sex steroids are intracellular, especially nuclear, and after puberty restricted to the hypothalamus, preoptic area and amygdala [16].

Adding to the "confusion" is the fact that until this century women rarely lived past childbearing age. In the United States, women's life expectancy did not extend past the age of 40 until the end of the first quarter of the last century, and in developing countries only in the last 50 years $[17,18]$. Thus, 3 million years of evolutionary selection in the human female has been concerned primarily with brain functions associated with "childbearing/rearing". The abundance of conflicting information as well as the fact that many women are completely asymptomatic (up to $40 \%$ ) [11] through midlife and natural menopause intuitively raises the possibility that there is a totally different mechanism producing "menopause," i.e. the MSC, thus requiring a totally different therapeutic approach. There is now substantial evidence to support the possibility of an age-related, brain circuitry change as the etiology for the MSC which may be, at least in part, genetically dependent with a primary genomic "clock" mechanism triggering dysfunctional (i.e. as defined by the abnormal behaviors listed) brain plasticity. And even in asymptomatic women, the "normal" aspects of this age-related plasticity mechanism may drive natural menopause [19].

\section{NEUROSCIENCE MYTHS OF THE LAST CENTURY}

Before further discussing the "menopause" problem, certain "neuroscientific myths" of the last century need to be dispelled. Firstly, the term "psychological" has no longer a primary meaning in that all behavior as well as behavioral interaction (verbal or otherwise) results from brain circuitry computational input/output involving mainly the limbic system [20]. Although the brain's limbic system has a well-established role in hormonal/sexual regulation, its major involvement is in cognitive, emotional, sleep and autonomic function. And therefore "psychological" manifestations depend on the presence of brain/limbic system activity, i.e. brain-dead individuals no longer suffer from neuroses, psychoses or personality disorders! Secondly the concept of a "biochemical imbalance" is also a simplistic holdover. The brain's concentration of neurotransmitters/modulators is a secondary phenomenon, depending on brain circuitry activation or inhibition and thus transmitter/modulator production/degradation, and this "biochemical quantity" is dependent on the activity of various brain circuits. The basis of this activity is complex as are the negative/positive (i.e. leading to health or disease) changes in circuitry which can occur over time that we term plasticity.

Neurotransmission refers to the electrochemical transmission at neuronal synapses. Neuromodulation is a slower process activated by transmitters and other compounds producing pre- and post-synaptic architectural changes as well as chemical changes at the neuronal membrane, all of which modify neurotransmission via membrane hyper- or hypopolarization. Brain plasticity refers to the ability of neuronal circuitry to reconfigure itself through architectural and/or physiochemical changes including new axonal and dendritic growth producing changes in synaptic site location; changes in type, density and sensitivity of synapses and/or receptors; changes in neurotransmitter/modulator rates of production/degradation; and changes in the structure/function of membrane receptor channels. Finally, it must be emphasized that each brain abnormality (disease state) has its 
very unique circuitry changes that only certain compounds specifically affecting those circuitry changes can positively affect, i.e. reverse the aberrant plasticity. Thus SSRIs are not a panacea for depression, but work only on those depressive "syndromes", i.e. circuitry dysfunctions, with primary involvement of serotonin receptors; and for example, appear to be of very little use in the MSC.

\section{4. "MENOPAUSE" AND ITS TREATMENT}

Since the middle of last century, women are living decades past reproductive prime migrating into previously biologically mandated male societal roles. This has made the MSC more visible defining this syndrome as a major medical as well as societal problem with major impact on the performance of afflicted individuals, reported to be $62 \%-84 \%$ of women [11]. The amount of the MSC caused job disruption especially in executives and professionals, as well as the MSC precipitated marital difficulties often leading to divorce with its major family disruption is impressive [21]. Since all components of the MSC can be mechanistically explained by "negative" changes in brain circuitry, or aberrant plasticity, the search for the "right" NT/MM or combination of such compounds (many of which were previously termed "psychotropics"), which can reverse this dysfunctional plasticity would be the obvious research direction for treatment of this widespread (probably epidemic) syndrome, i.e. the MSC.

Studies utilizing PET and Functional MRI will very likely be the definitive research path to define this aberrant plasticity which results in the specific circuitry producing the MSC. However after careful review of existing basic and clinical research, an open label, clinical investigation was undertaken. This trial pointed to a combination of a norepinephrine re-uptake inhibitor (with some effect on 5-HT and specifically the 5-HT2 receptors; and chronically reducing sensitivity and density of alpha-2 and beta-adrenergic receptors) and an alpha-1 noradrenergic/dopaminergic receptor blocker as the NT/MMs which can effectively reverse the MSC. 156 women with at least $80 \%$ of the MSC components $(8 / 10)$ listed in Section 2 were studied: 144 by retrospective analysis; 12 in a pilot clinical trial. NT/MMs used were perphenazine $2-8 \mathrm{mg} / \mathrm{d}$, with either amitriptyline $25-100 \mathrm{mg} / \mathrm{d}$ (4 - $6 \mathrm{mg} / \mathrm{d}$ perphenazine and $50-75 \mathrm{mg} / \mathrm{d}$ amitriptyline were utilized in $92 \%$ of patients), or later on, venlafaxine $75-150 \mathrm{mg} / \mathrm{d}$, given hs or divided bid, $1 / 3$ in the morning and $2 / 3$ at bedtime. Venlafaxine was especially useful in those women $(20 \%)$ where anticholinergic side effects and/or weight gain associated with amitriptyline were problematic. Initial treatment was administered for 4 - 6 months and tapered over one month. The following physiological parameters were monitored in the pilot clinical trial: bone density; FSH, total estrogen, cholesterol levels; and the MMPI (see Table 1).

Patient follow-up ranged between 2 and 12 years. The MSC onset range was 33 - 56 years and peaked between 35 - 45 years. The major presenting MSC symptoms were headache (44\% patients), fibromyalgia (36\%), and depression $(10 \%)$. Only $25 \%$ of subjects had a postmenopausal hormone profile on study entry. MSC remission under treatment occurred in 2 - 3 months, the known time required for circuitry plasticity to occur, and was usually complete. Symptom recurrence required repeated NT/MM courses. Recurrences were especially seen during the winter months, female limbic system circuitry being very sensitive to shortened daylight on probably an evolutionary basis related to seasonal reproduction. And sometimes chronic treatment was needed (almost always at lower doses than used for the initial treatment) in order to maintain the desired brain function, a state for which the individual became very aware. Side effects seen consisted of dry mouth and morning somnolence which were always transient. Acute or tardive dyskinesias never occurred. Basal ganglia tremor and/or hypertonia occurred rarely and only with high doses of perphenazine, and these symptoms cleared with dose reduction. Brain system inertia (Freud's "ego preservation instinct") was a major early treatment problem, i.e. a resistance to change of a behavioral pattern with its specific underlying brain circuitry pattern, even though this behavior was detrimental to the individual's optimal function.

Although classical control and blindness have not as yet been employed, the apparent universality and degree of response of all MSC components has implied a significant mechanistic intervention which warrants further investigational consideration.

This NT/MM combination has a definite salutary effect on hot flashes probably because this symptom manifestation involves hypothalamic thermoregulatory alpha-2 noradrenergic circuitry [22]. And reversing the abnormal behaviors of the MSC appears to have a positive effect on associated metabolic problems such as cardiac and cerebrovascular disease, and osteoporosis.

Table 1. Statistically significant results of monitored physiological parameters in the pilot clinical trial.

\begin{tabular}{ccc}
\hline Increased & Decreased & No change \\
\hline & MMPI measured & \\
psychopathology & \\
$(\downarrow$ Depression \& & Cholesterol \\
"Peak" & Total Estrogen \\
Bone density & Pathology; $\uparrow$ & FSH \\
& Appropriate & \\
& Mental Processes $)$ & \\
\hline
\end{tabular}


There may also be positive direct brain circuitry trophic effects, as well as brain-directed positive effects through the endocrinergic and immunologic systems once the aberrant limbic system circuitry is corrected. Of major note as previously discussed regarding the uniqueness of specific circuitry plasticity, these NT/MMs individually by themselves as well as SSRIs are not effective in the MSC. Occasionally, a morning SSRI has been added for additional serotonin effect; and alprazolam as needed for severe panic attacks. Even with chronic treatment, a case of tardive dyskinesia has never occurred in now 15 years of utilizing the alpha-1 noradrenergic/dopaminergic receptor blocker, again pointing to the uniqueness of each circuitry syndrome's aberrant plasticity. A similar situation exists with the basal ganglia circuitry syndromes, such that dopamine induced dyskinesias are common in Parkinsonism but are rarely seen in its variant, Progressive Supranuclear Palsy. Thus a PET scan based diagnostic signature for the MSC delineating this unique aberrant circuitry may be shortly obtainable when the proper radiotracer or combination of such ligands is identified.

As suggested by others, pre-menstrual syndrome, post-partum and post-hysterectomy depression may involve the same aberrant limbic system plasticity. Utilization of the above plasticity NT/MMs appears successful in these presentations, and those individuals with these presentations in earlier decades appear prone to the MSC $[23,24]$. Moreover, often the age of onset and severity of the MSC in a particular individual approximates that of their mother's suggesting at least some degree of genetic involvement $[23,25]$.

\section{CONCLUSION}

The MSC appears to be a genetically influenced, agerelated, genomically mediated example of aberrant brain/ limbic system plasticity which requires treatment attention, while menopause requires little if any intervention. "Menopause" reversal appears amenable to the utilization of NT/MMs which very specifically reverse the aberrant plasticity which has produced this dysfunctional circuitry and the resultant symptom complex. ERT/HRT and even SERMs have little, if any, affect on "menopause" i.e. the MSC.

\section{REFERENCES}

[1] Utian, W.H. (2005) The true symptoms associated with menopause confirmed after 33 years: Better late than never, but let's move on now. Menopausal Management, 10, 7-8.

[2] Pansini, F., Albertazzi, P., Bonaccorsi, G., et al. (1994) Trazadone: A non-hormonal alternative for climacteric symptoms. Menopause, 1, 159-160. doi:10.1097/00042192-199400130-00046
[3] Loprinzi, C.L., Sloan, J.A., Perez, E.A., et al. (2002) Phase III evaluation of fluoxetine for treatment of hot flashes. Journal of Clinical Oncology, 6, 1578-1583. doi:10.1200/JCO.20.6.1578

[4] Thal, L.J., Thomas, R.G., Mulnard, R., et al. (2003) Estrogen levels do not correlate with improvement in cognition. Archives of Neurology, 60, 209-212. doi:10.1001/archneur.60.2.209

[5] Popat, R.A., Van Den Eden, S.K., Tanner, C.M., et al. (2005) Effect of reproductive factors and postmenopausal hormone use on the risk of Parkinson disease. Neurology, 65, 383-390. doi:10.1212/01.wnl.0000171344.87802.94

[6] Riggs, B.L. and Melton III, L.J. (1986) Involutional osteoporosis. New England Journal of Medicine, 314, 1676-1686. doi:10.1056/NEJM198606263142605

[7] Kobchitt, L., Chompootweep, S., Chaikittisilpa, S., et al. (1995) Climacteric complaints of Thai women in various social classes. Menopause, 2, 278. doi:10.1097/00042192-199502040-00111

[8] Kasuga, M., Makita, K., Ishitani, K., et al. (2004) Relation between climacteric symptoms and ovarian hypofunction in middle-aged and older Japanese women. Menopause, 11, 631-638. doi:10.1097/01.GME.0000119984.87302.30

[9] Schnatz, P.F., Serra, J., O'Sullivan, D.M., et al. (2004) Menopausal symptoms in Hispanic women and the role of socioeconomic factors. Menopause, 11, 694.

[10] Hays, J., Ockene, J.K., Brunner, R.L., et al. (2003) Effects of estrogen plus progestin on health-related quality of life. New England Journal of Medicine, 348, 18391854. doi:10.1056/NEJMoa030311

[11] Woods, N.F. (1992) Menopausal distress: A model for epidemiologic investigation. In: Voda, A.M., Dinnistein, M. and O'Donnell, S.R., Eds., Changing Perspective on Menopause, University of Texas Press, Austin, 220-238.

[12] Lee, K.A. and Taylor, D.L. (1996) Is there a generic midlife woman? The health and symptom experience of employed midlife women. Menopause, 3, 154-164. doi:10.1097/00042192-199603030-00007

[13] Rasgon, N., Shelton, S. and Halbreich, U. (2005) Perimenopausal mental disorders: Epidemiology and phenomenology (Review). CNS Spectrums, 10, 471-478.

[14] Pearlstein, T.B. (1995) Hormones and depression: What are the facts about premenstrual syndrome, menopause and hormone replacement therapy? American Journal of Obstetrics and Gynecoogy, 173, 646-653. doi:10.1016/0002-9378(95)90297-X

[15] Sherwin, B.B. (1996) Hormones, mood, and cognitive functioning in postmenopausal women. Obstetrics and Gynecology, 87, 20S-26S. doi:10.1016/0029-7844(95)00431-9

[16] Soares, C.N., Prouty, J., Born, L. and Steiner, M. (2005) Treatment of menopause-related mood disturbances (Review). CNS Spectrums, 10, 489-497.

[17] Spiegelman, M., and Erhardt, C.L. (1974) Mortality and longevity in the United States. In: Erhardt, C.L., Ed., Mortality and Morbidity in the United States, Harvard University Press, Cambridge, 1-20. 
[18] Department for Economic and Social Information and Policy Analysis (1994) 1992 Demographic Yearbook. United Nations Press, New York.

[19] Wise, P.M., Kashon, M.L., Krajnak, K.M., et al. (1997) Aging of the female reproductive system: A window into brain aging. Record of Progress in Hormone Research, 52, 279-305.

[20] Jaffe, S.L. (2000) Neurology and psychiatry: Closing the great divide. Neurology, 55, 603-604.

[21] Woods, N.F. and Mitchell, E S. (2011) Symptom interference with work and relationships during the menopausal transition and early postmenopause: Observations from the Seattle Midlife Women's Health Study. Menopause, 18, 654-661. doi:10.1097/gme.0b013e318205bd76

[22] Freedman, R.R., Woodward, S. and Sabharwal, S.C. (1990) Alpha-2-adrenergic mechanism in menopausal hot

\section{ABBREVIATIONS}

MSC (menopausal symptom complex);

ERT/HRT (estrogen replacement therapy/hormone replacement therapy);

NT/MM (neurotransmission-modulation modifier);

SSRI (selective serotonin reuptake inhibitor);

PET (positron emission tomography); flushes. Obstetetrics and Gynecology, 76, 573-578.

[23] Feld, J., Halbreich, U., and Karkun, S. (2005) The association of perimenopausal mood disorders with other reproductive-related disorders (Review). CNS Spectrums, 10, 461-470.

[24] Freeman, E.W., Samuel, M.D., Rinaudo,P.J. and Sheng, L. (2004) Premenstrual syndrome as a predictor of menopausal symptoms. Obstetrics and Gynecology, 103, 960-966. doi:10.1097/01.AOG.0000124804.81095.7f

[25] Skarsgard, C., Bjors, E., Nedstrand, E., et al. (1996) Do women's premenstrual symptoms and their mother's climacteric history predispose to their own vasomotor symptoms? Menopause, 3, 133-139.

MRI (magnetic resonance imaging);

HT (hydroxytryptamine);

FSH (follicle stimulating hormone);

MMPI (Minnesota Multiphasic Personality Inventory;

SERM (selective estrogen receptor modulator). 